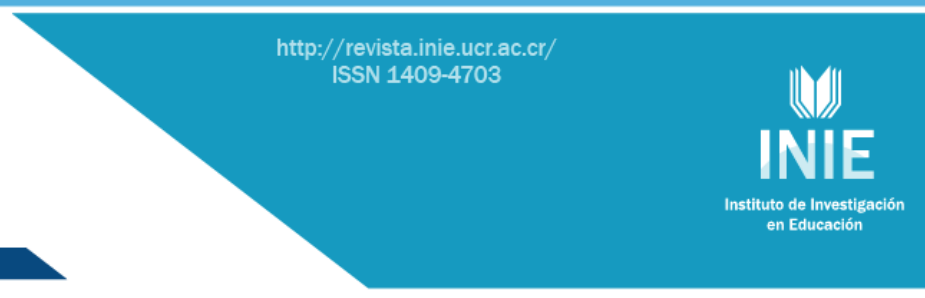

\title{
EL NIVEL EDUCATIVO Y SOCIOECONÓMICO DEL HOGAR Y HABILIDADES PRE-LECTORAS EN ESCUELAS URBANO Y URBANO-MARGINALES DE LA PROVINCIA DE MENDOZA- ARGENTINA
}

EDUCATIONAL AND SOCIOECONOMIC LEVEL OF PARENTS AND PRE-READING SKILLS FROM URBAN AND URBAN-MARGINAL SCHOOLS FROM MENDOZAARGENTINA

\author{
Volumen 16, Número 2 \\ Mayo-Agosto \\ pp. $1-26$
}

\author{
Yanina Canales Jara \\ María Elsa Porta
}

Revista indizada en REDALYC, $\underline{\text { SCIELO }}$

Revista distribuida en las bases de datos:

LATINDEX, DOAJ, E-REVIST@S, IRESIE, CLASE, DIALNET, SHERPA/ROMEO, QUALIS, MIAR

Revista registrada en los directorios:

ULRICH'S, REDIE, RINACE, OEI, MAESTROTECA, PREAL, CLACSO 


\title{
EL NIVEL EDUCATIVO Y SOCIOECONÓMICO DEL HOGAR Y HABILIDADES PRE-LECTORAS EN ESCUELAS URBANO Y URBANO- MARGINALES DE LA PROVINCIA DE MENDOZA-ARGENTINA EDUCATIONAL AND SOCIOECONOMIC LEVEL OF PARENTS AND PRE-READING SKILLS FROM URBAN AND URBAN-MARGINAL SCHOOLS FROM MENDOZA- ARGENTINA
}

\author{
Yanina Canales Jara ${ }^{1}$ \\ María Elsa Porta²
}

\begin{abstract}
Resumen: En Argentina, las dificultades en la adquisición de la lectura se originan en diferencias lingüísticas asociadas al nivel sociocultural donde se desarrollan los niños. El objetivo de este artículo es analizar, en escolares de 4 y 5 años, la relación entre el nivel educativo y socioeconómico del hogar con habilidades lingüísticas precursoras de la lectura. Esta es una investigación de carácter cuantitativo y de profundidad comparativa. Se evaluaron 120 niños y niñas de salas de 4 y 5 años de Jardín de Infantes de escuelas urbanas $(n=60)$ y urbano-marginales $(n=60)$. El nivel socioeconómico del hogar y educativo de los padres se calculó a partir de datos de los registros escolares. En los niños y niñas se evaluó el nivel de vocabulario, conocimiento del nombre y sonido de la letra y conciencia fonológica. Se observó un efecto significativo de la zona sobre el nivel educativo de los padres y el socioeconómico. Se obtuvieron correlaciones positivas del nivel socioeconómico y educativo con el conocimiento del nombre y sonido de la letra. Ambas variables contextuales explicaron parte de la varianza en el conocimiento del nombre y sonido de las letras en niños y niñas de 4 años únicamente: 6\% y $13 \%$ respectivamente. Los resultados sugieren que las variables contextuales influyen en el conocimiento alfabético pre-escolar que presentan los niños y las niñas y que la intervención pedagógica recibida en la escuela promueve el desarrollo de habilidades lingüísticas. Frente a contextos socioeconómicos heterogéneos, la educación escolar es una variable protectora que regula las desigualdades de orden social promoviendo el desarrollo de habilidades lingüísticas precursoras de la lectura al inicio de la escolaridad.
\end{abstract}

Palabras clave: NIVEL SOCIOECONÓMICO FAMILIAR, NIVEL EDUCATIVO, HABILIDADES LINGÜÍSTICAS, HABILIDADES PRELECTORAS, ESCOLARES DE 4 Y 5 AÑOS.

\begin{abstract}
In Argentina, reading acquisition difficulties come from the linguistic differences, associated to the sociocultural context in which children grow. The goal of this paper is to analyze, in 4 and 5 years old children, the relationship between the socioeconomic and educational home level and the precursor linguistic abilities of reading. This is a quantitative and comparative research. We evaluated 120 children from 4 and 5 years-old classroom from urban $(n=60)$ and urban-marginal schools $(n=60)$. We calculated the parental socioeconomic and educational level from data gathered from the school records. We assessed children's vocabulary, letter-name and sound knowledge and phonological awareness skills. It was observed a significative effect of the area (urban and urban-marginal) over the parental socioeconomic and educational level. We observed positive relationship between both the socioeconomic and educational level and the letter-name and sound knowledge variable. The regression analysis showed that only in the 4 year old group the contextual variables explained some of the children vocabulary and letter-name and sound knowledge variance: $6 \%$ and $13 \%$, respectively. The results suggest that the contextual variables influence the letter-name and sound knowledgeability level that children show at the pre-school stage and that the pedagogical intervention received in school promotes linguistic skills. Facing heterogeneous socioeconomic contexts, school education is a protective variable that regulates inequalities due to sociocultural differences promoting linguistic abilities precursors of reading.
\end{abstract}

Key Words: SOCIOECONOMIC STATUS, FAMILY EDUCATIONAL LEVEL, LINGUISTIC SKILLS, PREREADING SKILLS, 4 AND 5 YEAR OLD SCHOOL-CHILDREN

1 Catedrática en la Universidad del Aconcagua, Mendoza, Argentina. Licenciada en Fonoaudiología. Dirección electrónica: naniyacci@hotmail.com

2 Catedrática en la Universidad del Aconcagua, Mendoza, Argentina; Investigadora Adjunta del Consejo Nacional de Investigaciones Científicas y Técnicas, Instituto de Lingüística, Universidad Nacional de Cuyo, Argentina. Doctora en Educación. Dirección electrónica: mariaelporta@yahoo.com.ar

Artículo recibido: 18 de junio, 2015

Enviado a corrección: 26 de noviembre, 2015

Aprobado: 7 de marzo, 2016

Volumen 16 Número 2, Año 2016, ISSN 1409-4703 


\section{Introducción}

El presente artículo analiza la relación existente entre las habilidades lingüísticas de preescolares mendocinos y mendocinas de 4 y 5 años de edad que asisten a escuelas urbanas y urbano-marginales y el nivel socioeconómico y educativo del contexto familiar en el que crecen. Según estudios longitudinales de corte empírico en psicopedagogía el aprendizaje de la lectura se inicia con el lenguaje y por ese motivo los predictores más significativos del futuro rendimiento lector son de origen lingüístico (Dickinson y Tabors, 2001). Entre las habilidades lingüísticas prelectoras más citadas en las investigaciones realizadas en niños preescolares se encuentran la conciencia fonológica, el conocimiento del nombre y sonidos de las letras y el vocabulario (por ejemplo, Baker, Al Otaiba, Ortiz, Correa y Cole, 2014; Ehri, Nunes, Willows, Shcuster, Yaghoub-Zadeh y Shanahan, 2001).

Desde la perspectiva del modelo contextual de Bronfenbrenner (1995) es posible apreciar cómo factores contextuales de la vida del niño o la niña previos al inicio de su escolaridad, tales como el nivel socioeconómico y educacional de la familia, influyen en el desarrollo de sus habilidades lingüísticas. Aunque las variables contextuales no han sido identificadas como predictores principales del futuro rendimiento lector, se ha observado consistentemente que aquellos niños y niñas que crecen en ambientes más favorecidos presentan un mayor desarrollo de habilidades lingüísticas que el grupo de infantes provenientes de sectores menos favorecidos. Se ha demostrado que el nivel socioeconómico, a través de mecanismos mediadores tales como la salud y nutrición de la persona menor, el ambiente en el hogar, la salud mental de los padres y la interacción familiar, interfiere en el desarrollo cognitivo y del lenguaje (por ejemplo, Borzone, Rosember, Diuk y Amado, 2005; Dickinson y Tabors, 2001; Filippetti, 2012). Con respecto al nivel educativo de los padres se ha observado que quienes tienen un nivel de educación superior recrean situaciones de comunicación más ricas y estimulantes para sus hijos e hijas, promoviendo el desarrollo de distintos aspectos del lenguaje tales como el vocabulario o la comprensión oral (Filippetti, 2012; Hoff y Tian, 2005). En consecuencia, estudios dirigidos a identificar predictores principales de la adquisición de la lecto-escritura, tales como el ambiente literario en el hogar, el nivel de vocabulario o la conciencia fonológica -entre otrosconsideran como variables de control para otorgar rigor científico a sus estudios el nivel educativo y socioeconómico del hogar donde se desarrollan los niños y las niñas (por ejemplo, Dickinson, Anastasopoulos, McCAbe, Peisner-Feinberg y Poe, 2003). 
Como mencionamos, existen evidencias sobre las diferencias lingüísticas que presenta la niñez al inicio de la escolaridad, asociadas al nivel educativo de los padres y al socioeconómico del hogar. Sin embargo, aunque algunas investigaciones consideran la zona escolar como un indicador del estado de las variables contextuales mencionadas, escasos estudios analizan la relación existente entre ambas variables contextuales (nivel educativo y socioeconómico), la zona escolar a la que asisten los participantes y las habilidades lingüísticas consideradas precursoras del proceso de adquisición de la lectura tales como el vocabulario, la conciencia fonológica y el conocimiento del nombre y sonido de las letras.

Por ello, es objetivo del presente trabajo responder a los siguientes interrogantes: ¿existen diferencias en el nivel educativo y socioeconómico de los padres de preescolares que estén asociadas a la zona en la que está ubicada la escuela a la que asisten sus hijos e hijas?; ¿existe relación entre el nivel educativo de los padres, el nivel socioeconómico y las habilidades lingüísticas que evidencian preescolares?; ¿existe relación entre el nivel educativo, socioeconómico y las habilidades lingüísticas en niños y niñas que asisten a Escuelas Urbanas?; ¿existe relación entre el nivel educativo, socioeconómico y las habilidades lingüísticas en niños y niñas que asisten a Escuelas Urbano Marginales?

\section{Referente Teórico}

\subsection{El modelo comprehensivo del aprendizaje lingüístico}

El modelo contextual de Bronfenbrenner (1995) ofrece un marco teórico adecuado para comprender cómo factores contextuales tales como el nivel socioeconómico o educativo familiar se relacionan con las habilidades cognitivas que desarrolla el niño previo al inicio de la escolaridad y que formarán parte del proceso de reconocimiento de las palabras escritas. El mismo sostiene que la persona en desarrollo se encuentra rodeada de una serie de sistemas complejos que interactúan entre sí: el microsistema, el mesosistema, el exosistema y el macrosistema. En el microsistema se distinguen factores internos y externos. Los primeros aluden a las estrategias cognitivas de identificación de palabras y a los mecanismos neurológicos intervinientes.

Según Bravo Valdivieso (2000) los procesos cognitivos necesarios para aprender a leer deben considerarse en interacción con factores emocionales, socioculturales y metodológicos. Por ello el modelo comprehensivo del aprendizaje lingüístico inicial se vertebra en torno a la perspectiva contextual de Bronfenbrenner (1995). Desde esta perspectiva en el aprendizaje lingüístico inicial los factores externos están representados por 
la familia y las personas del ambiente inmediato del niño o niña; es decir, la estructura social próxima en la que se inicia su proceso de socialización. Tres dimensiones hogareñas revisten importancia para la adquisición de la lectura: el nivel académico de los padres y el ambiente literario. Por otra parte, en el mesosistema incluimos las instituciones y agentes que -al interactuar con la niñez- facilitan el aprendizaje lingüístico inicial. Entre ellos destacamos la escuela, las creencias del docente, sus estrategias de interacción verbal, el curriculum y el ambiente literario áulico. Finalmente, como variables integrantes del exosistema y del macrosistema, se toman en cuenta aspectos socio-culturales tales como el nivel socioeconómico familiar, la transparencia del idioma y las políticas educativas implementadas para la enseñanza de la lectura (Porta e Ison, 2011).

\subsection{Habilidades lingüísticas precursoras de la lectura}

Desde la perspectiva cognitiva la destreza lectora se concibe como una habilidad compleja que implica varios procesos y recursos perceptivos, cognitivos y lingüísticos. Aún más, estudios longitudinales evidencian que la lectura se inicia con el lenguaje y que por ese motivo los predictores más significativos del futuro rendimiento lector son de origen lingüístico (por ejemplo, Dickinson y Tabors, 2001).

Entre las habilidades lingüísticas prelectoras más citadas en las investigaciones realizadas en niños y niñas de Jardín de Infantes se encuentran la conciencia fonológica, el conocimiento del nombre y sonidos de las letras y el vocabulario. Con respecto a su valor predictivo, podemos mencionar que mientras que la habilidad de conciencia fonológica y el conocimiento del sonido de las letras contribuyen con la identificación de palabras escritas poco frecuentes en primer y segundo grado (Ehri et al., 2001; Porta y Difabio 2012), el vocabulario y la comprensión oral de oraciones favorecen los procesos de comprensión lectora en grados ulteriores (Anderson y Freebody, 1981; Dickinson y Tabors, 2001, entre otros).

La conciencia fonológica, habilidad para identificar y manipular las unidades fonológicas de las palabras tales como fonemas, sílabas y rimas (Ziegler y Goswami, 2005), ha sido identificada como el principal predictor de la lectura de palabras nuevas. La correlación positiva y significativa entre los logros en el aprendizaje de la lectoescritura y la competencia en conciencia fonológica ha sido ampliamente comprobada (por ejemplo, Bradley y Bryant, 1983; Porta, Harper y Kraft, 2010). Aun más, la conciencia fonológica puede estimularse y desarrollarse generando efectos positivos en el nivel de rendimiento 
lector. De allí que numerosas investigaciones evalúan los efectos de programas de intervención sobre el futuro nivel lector, demostrando el poder predictivo de dicha habilidad en lenguas con relativa transparencia idiomática (Ehri et al., 2001).

El conocimiento del nombre de la letra es otro factor de importancia por tener en cuenta en la evaluación del nivel de habilidades lingüísticas con el que arriban los niños y las niñas al ámbito escolar. En la práctica cotidiana observamos a menudo un énfasis en la enseñanza de los nombres de las letras; sin embargo, se ha observado que durante la etapa pre-escolar, identificar los sonidos de algunas letras del alfabeto facilita la comprensión del principio alfabético, es decir, comprender que son los sonidos del habla los que están representados en los grafemas del abecedario. En este sentido, el conocimiento del nombre de la letra es otro predictor potente, junto con la conciencia fonémica (Share, Jorm, Maclean y Mathews, 1984). Muter, Hulme, Snowling y Taylor (1998) en un estudio longitudinal realizado durante los dos primeros años de escolaridad, encontraron que el conocimiento del nombre de la letra predijo tanto el nivel de lectura como el de escritura. Por lo tanto, la habilidad para establecer conexiones entre fonemas y las palabras que los representan facilita el aprendizaje de la lectura (Adams, Foorman, Lundberg y Beeler, 2003).

Por otra parte, el nivel de vocabulario que presenta cada infante al iniciar su escolaridad es un predictor significativo del nivel de comprensión lectora (Anderson y Freebody, 1981; Dickinson y Tabors, 2001; Dickinson, 2012). Un estudio longitudinal llevado a cabo en escuelas primarias demostró que: a) el nivel de vocabulario de la niñez al iniciar la escuela predice el nivel de decodificación de palabras escritas y comprensión lectora en primer grado; b) a partir de segundo grado, el nivel lector influye sobre el nivel de vocabulario en dicho grado y en los grados ulteriores y c) existe una relación recíproca entre un vocabulario avanzado y el nivel lector alcanzado en etapas ulteriores de la escuela primaria (Verhoeven, van Leeuwe y Vermeer, 2011).

La fuerza predictiva del nivel de vocabulario sobre la lectura se debe a que el sistema de escritura representa la estructura fonológica del habla: para aprender a leer la persona menor inicialmente debe identificar los sonidos de las palabras para luego asociar dichos sonidos a las letras correspondientes. Por ello las conexiones fonológicas que establezca antes de la enseñanza formal de la lectura juegan un rol crucial en el proceso de identificación de las palabras escritas. Particularmente, el grado de especificidad con la cual las estructuras sonoras de las palabras son representadas en el léxico mental influye en la conciencia fonémica y otras habilidades lingüísticas. En otras palabras, niños y niñas con un 
elevado nivel de vocabulario presentan representaciones fonológicas más claras de las palabras y esto contribuye tanto al desarrollo de la conciencia fonológica como de la lectura. En relación con la comprensión lectora, estudios previos demuestran que la automatización adquirida en la identificación de palabras escritas es esencial tanto para el desarrollo del vocabulario como el de la comprensión lectora. El estudio de Verhoeven, van Leeuwe, y Vermeer (2011) evidencia de manera empírica que en lenguas con mayor transparencia idiomática, como es el caso del alemán o del español, el nivel de vocabulario de la persona menor facilita la comprensión lectora desde los estadios iniciales del aprendizaje de la lectura.

\subsection{Influencia del nivel educativo de los padres en el desarrollo de habilidades lingüísticas}

Según un informe de la Unesco (2007) el retraso escolar temprano se encuentra asociado a diferentes experiencias socioculturales y lingüísticas a las que están expuestos los niños y las niñas en sus hogares y que se manifiestan en la lecto-escritura. De este modo, el nivel educativo de los padres es considerado una variable a controlar en estudios dirigidos a evaluar predictores significativos del rendimiento lector en las etapas iniciales de adquisición de la lectura por la relación que guarda con el nivel de habilidades lingüísticas que presenta la niñez al inicio de la escolaridad (Dickinson et al., 2003; Dickinson y Tabors, 2001). Mientras que el bajo nivel educativo de los padres influye negativamente en el rendimiento escolar de niñas y niños, se ha observado que padres con un nivel educativo superior promueven ambientes ricos en estímulos lingüísticos y cognoscitivos. Madres con educación superior emplean un vocabulario más variado y leen más a sus hijos que madres con una educación equivalente al secundario, lo cual genera diferencias en el nivel de vocabulario que presentan sus hijos e hijas (Hoff, 2003). Aun más, el nivel de los padres influye en habilidades preacadémicas, lingüísticas y académicas y en la actitud que los escolares adoptan frente a la educación (Benítez, Vargas, Hernández, Sánchez y García, 2007; Dearing, McCartney, Weiss, Kreider y Simpkins, 2004). La educación de los padres es una variable que media a través de factores tales como los "hábitos de vida, los modelos de interacción, la comunicación lingüística en el hogar y las expectativas educacionales para los hijos" (Jadue, 1997, p.76). En su revisión Jadue señala que el nivel educativo influye a través de factores tales como la disponibilidad en el hogar de textos y materiales de apoyo a la tarea escolar, así como también la manera en que se los utiliza. Asimismo influye en la 
calidad y cantidad de estrategias de aprendizaje que ayuden a los niños a tener éxito en la escuela (Jadue, 1997, p.77).

Con respecto a las experiencias lingüísticas que comparten los padres y las personas menores en el hogar, estudios que evalúan la influencia del ambiente sobre el aprendizaje inicial de la lectura señalan que las actividades literarias tales como la lectura de cuentos y conversaciones extendidas, presentan un efecto inmediato y duradero sobre el nivel lector de los niños (Dickinson y Tabors, 2001; Porta, 2008; Porta y Difabio, 2011). Parte del conocimiento sobre la escritura se adquiere desde la etapa preescolar en función de las experiencias literarias que haya tenido el niño y se refiere al conocimiento de las letras, las palabras escritas y la forma en que los libros están organizados. En particular, se ha demostrado que los primeros conocimientos que los niños adquieren sobre el nombre y sonido de las letras del alfabeto se deben a la enseñanza explícita por parte de los adultos mediante actividades organizadas en el hogar (Burgess, Hecht y Lonigan, 2002).

Algunas actividades específicas que contribuyen con la adquisición de este conocimiento previo sobre el sistema de escritura son la distinción entre los dibujos y la escritura, comprender que las palabras representan significados, leer de izquierda a derecha y conocer la forma en que se sostiene un libro y se voltean sus páginas. Dickinson y Tabors (2001) visitaron las casas de los niños evaluados en distintos aspectos del lenguaje y las clases a las que asistían, registrando las interacciones verbales que acaecían entre las niñas y los niños con sus padres y docentes. Los autores señalan que ambientes ricos en experiencias lingüísticas y literarias tanto en la escuela como el hogar durante la etapa preescolar pueden generar cambios dramáticos tanto en el desarrollo del lenguaje como en la adquisición de la lectura.

\subsection{Influencia del nivel socioeconómico en el nivel de rendimiento en la ejecución de tareas lingüísticas}

Con respecto al nivel socioeconómico, un significativo corpus de estudios analiza su relación con el rendimiento escolar y la habilidad cognitiva general de los escolares así como también con el desarrollo de habilidades lingüísticas. La repitencia y el abandono escolar afectan principalmente a niñas y niños provenientes de sectores socio-económicos menos favorecidos: mientras que entre quienes provienen de nivel socio-económico bajo, 23 de cada 100 repiten, los que crecen en entornos más favorecidos repiten 4,5 de cada 100 (Unicef, 2007). Importa aquí aclarar que el fracaso escolar es el resultado de una serie de 
circunstancias que interfieren en la plena escolarización de los niños y las niñas, tales como no entender los temas de la clase, faltar mucho, no contar con útiles o libros suficientes para estudiar o no tener a quien recurrir por más apoyo entre otras.

Los estudios de Buckner, Bassuk y Weinreb (2001) reportan que la condición socioeconómica es predictora del bajo aprovechamiento escolar, ya que la niñez de nivel socioeconómico bajo evidencia dificultades académicas y de habilidad intelectual en general. Jadue (1997) identifica como variables mediadoras entre el nivel socioeconómico y la habilidad cognitiva general las características de los hogares de bajo NSE. La autora sostiene que éstas influyen adversamente en el desarrollo cognitivo y sicosocial de los niños y las niñas, limitando su experiencia cognitiva. Esto puede explicar parcialmente la desventaja que el alumnado de entornos socioeconómicos más desfavorecidos presenta en relación a sus pares provenientes de clase media en tareas en las que el desarrollo cognitivo alcanzado interactúa con las estrategias de aprendizaje, en especial de la lecto-escritura, en la que gran parte de los conocimientos básicos requeridos para su adquisición se desarrollan en los años previos al inicio de la escolaridad. El hacinamiento repercute en los estilos de interacción familiar afectando la concentración, la memorización y la correcta discriminación de estímulos. El ruido ambiental en estrecha relación con el hacinamiento, interfiere con hábitos de estudio tales como sentarse, fijar la atención, mirar figuras, escuchar una historia o un cuento, ejercitar el "por qué", lo que resulta en una habilidad discriminativa perceptual deficiente, escaso desarrollo del lenguaje y atención poco sostenida. Por otra parte, a pesar del reconocimiento del valor de la educación por parte de los padres, en contextos socioeconómicos adversos las interacciones entre padres e hijos/hijas con respecto al aprendizaje suelen estar acompañadas de estrategias poco efectivas o actitudes limitadas a las demandas de los docentes. De este modo, forman parte del constructo relacionado con la capacidad de aprendizaje de la niñez. Las madres de bajo NSE se reportan a sí mismas como pasivas y acomedidas frente a las consignas de los docentes mientras que las de clase media lo hacen como activamente involucradas en las tareas escolares de sus hijos (Majluf citado por Jadue, 1997).

Las dificultades observadas en el aprendizaje inicial de la lectura se deben, en su mayoría, a diferencias lingüísticas que presentan los escolares al inicio de la vida escolar, entre ellas habilidades consideradas precursoras de la lectura tales como la habilidad de conciencia fonológica, conocimiento del nombre y de la letra y conocimiento de correspondencias entre el sonido y las letras entre otras (Borzone et al., 2005). Estudios que 
analizan las diferencias lingüísticas relativas al proceso de alfabetización en niños y niñas provenientes de distintos niveles socio-económicos, sugieren que las mismas están relacionadas con el nivel de alfabetización del entorno familiar y social en el que crecen las personas menores (Borzone et al., 2005; Dickinson y Tabors, 2001). En este sentido, el estudio realizado por Borzone et al. (2005), en el que se evaluaron habilidades lingüísticas relativas al proceso de alfabetización en niños de distintos niveles socioeconómicos, se observó que los niños y las niñas provenientes de niveles más desfavorecidos obtuvieron respuestas menos avanzadas en las pruebas formales de lenguaje, lectura y escritura que aquellos registrados en otros sectores. Con respecto al vocabulario, el estudio de Hart y Risley (1995) reporta que el número de palabras que conocen los niños y las niñas de nivel socioeconómico bajo es dos veces inferior al que conocen quienes provienen de niveles socioeconómicos más altos.

Sin embargo, desde una perspectiva esperanzadora, todo retraso escolar temprano puede prevenirse, ya sea mediante eventos de capacitación para las familias para promover ambientes ricos en actividades literarias en el hogar o atenuarse mediante la intervención pedagógica específica en el ámbito escolar (por ejemplo, Dickinson y Caswell, 2007; Ehri et al., 2001).

\section{Metodología}

\subsection{Muestra}

Los muestra estuvo constituida por niños de 4 y 5 años de ambos sexos, de Jardines Urbanos y Urbano-Marginales estatales de la provincia de Mendoza. También se obtuvieron datos relativos al nivel socioeconómico y educativo de los respectivos padres de los registros escolares.

Se evaluó un total de 120 niños y niñas: 60 escolares de 4 años ( $n=30$ de escuelas Urbanas, de los cuales 9 son varones y 21 niñas; y 30 niños de escuelas urbanomarginales, de los cuales 15 son varones y 15 niñas); y 60 de 5 años ( $n=30$ de escuelas urbanas: de los cuales 15 son varones y 15 niñas; y $n=30$ de escuelas urbano-marginales: de los cuales 15 también son varones y 15 niñas). Los escolares de sala de 4 con edades comprendidas entre 4 años y 2 meses a 4 años y 11 meses $(E M=55.1$; DT = 2.7) y de sala de 5 años con edades comprendidas entre 5 años y un mes a 5 años y 11 meses $(E M=65.6$; DT $=2.8), p<$ 0.000. Todas las personas participantes presentaban un rendimiento escolar acorde a la mayoría de sus pares, según informó la docente. Se esperaba que la muestra estuviese 
formada por niños y niñas hispanohablantes que presentaran las siguientes características: a) el español como primera lengua; b) de nivel intelectual acorde con la mayoría de los niños y niñas de la misma edad cronológica; c) sin alteraciones neurológicas ni deficiencias auditivas; d) con ausencia de dificultades del lenguaje y del aprendizaje; e) de nivel socioeconómico bajo y e) que aún no decodificaban fonéticamente palabras escritas (Tabla 1).

\subsection{Procedimientos y mediciones}

Luego de obtener las autorizaciones pertinentes de las direcciones y de los padres de las personas menores participantes, las docentes informaron sobre el rendimiento escolar de estas. El grupo de escolares fue evaluado al comienzo del ciclo lectivo en el establecimiento educativo y las pruebas de conciencia fonológica fueron administradas en forma individual en una habitación silenciosa que les permitiera a las niñas y los niños identificar con claridad las tareas de evaluación que implicaran habilidades auditivas. Se obtuvieron mediciones de variables lingüísticas y ambientales:

\subsubsection{Medición de Variables lingüísticas}

a. Vocabulario. Se administró el subtest del WIPPSI (Wechsler, 2004). El mismo presenta una excelente fiabilidad (0.91) y correlaciona significativamente con la escala completa que abarca un amplio rango de edad (0.86).

b. Conocimiento del nombre y sonido de la letra. Se presentan las letras del abecedario en tipografía grande, imprenta mayúscula; cada letra en tarjeta independiente. Se le pregunta al niño o la niña si conoce el nombre de la letra, el sonido y si puede decir el nombre de algo o alguien que empiece con el sonido de la letra. En la presente evaluación, se tabularon las tres categorías por separado (nombre de la letra, sonido y palabra que empiece con dicho sonido). Se otorga 1 punto por cada respuesta correcta. Se sumó el puntaje obtenido en cada categoría para obtener una puntuación total.

c. Conciencia Fonológica. Descripción de las tareas. La selección de las tareas para la evaluación de las habilidades fonológicas se realizó en función de los siguientes criterios: (1) Que se hubiera informado la validez de su valor predictivo en relación al rendimiento lector y que dicho valor fuera significativo; (2) que abarcara las dos dimensiones que caracterizan a las habilidades de conciencia fonológica -la demanda de la tarea (análisis, síntesis y manipulación) y el tamaño de las unidades fonológicas (sílaba, subsílabas y fonemas)-; (3) 
que las habilidades fueran evolutivamente apropiadas para los niños a fin de evitar los efectos de piso y techo (Fernández Cano, Machuca y Lorite, 2002; Pennington y Lefly, 2001; Yopp, 1988).

c.1. Tareas que evalúan las distintas unidades fonológicas y que difieren en la demanda de la misma

1. Síntesis de Sonidos (Woodcock y Muñoz-Sandoval, 1996). Esta tarea consiste en que el participante escuche de un grabador palabras segmentadas en sílabas y/o fonemas que luego debe integrar en la palabra correspondiente. Evalúa la segmentación de palabras en sus distintas unidades fonológicas: sílabas y fonemas. Por orden de complejidad las palabras grabadas están segmentadas primeramente en sílabas; luego en combinaciones de sílabas y fonemas; finalmente en fonemas únicamente. La prueba consta de 33 ítems. Se otorga un punto por cada respuesta correcta. Se suspende la prueba luego de 6 respuestas consecutivas incorrectas. Esta tarea presenta una media de confiabilidad (como consistencia interna) de 0.86 para las edades comprendidas entre los 5 y 19 años.

2. Segmentación Silábica. Contar las sílabas en una palabra (Jiménez González y Ortiz González, 1995). Consiste en segmentar palabras bisilábicas y trisilábicas en sus correspondientes sílabas. Las palabras se van presentando de una en una. Se otorga 1 punto por cada respuesta correcta. Esta tarea presenta una confiabilidad de 0.88.

3. Aislar sílabas y fonemas en palabras (Jiménez-González y Ortiz-González, 1995) consiste en buscar -en series de dibujos- aquellos cuyos nombres contienen: primero, el fonema vocálico emitido por el examinador en posición inicial (por ej., /i/ en dibujos de un indio, reloj, pipa y canilla); segundo, la sílaba en posición inicial (por ej., /sa/ en dibujos de saco, tambor, barca, luna) y final (por ej. /na/ en dibujos de saco, tambor, barco, luna); tercero, el fonema consonántico en posición inicial (por ej., /f/ en dibujos de foca, gallina, tractor, dado) y final (por ej., /r/ en dibujos de foca, gallina, tractor, dado). De esta prueba se obtiene puntuación para los siguientes factores:

Aislar sílabas (confiabilidad: 0.51). Se refiere a la habilidad para detectar aquellos dibujos cuyos nombres comienzan o terminan por un sonido vocálico o sílaba determinada.

Aislar sonidos consonánticos (confiabilidad: 0.52). Se refiere a la habilidad para detectar aquellos dibujos cuyos nombres comienzan o terminan por un sonido consonántico.

4. Omisión de sílabas y fonemas en las palabras (Jiménez-González y Ortiz-González, 1995). Consiste en ir nombrando series de dibujos omitiendo: primero, el fonema vocálico 
inicial -por ej., dibujos de oveja (veja); uva (va)-; segundo, la sílaba inicial -por ej., boca, (ca); pino (no)- y tercero, la sílaba final -por ej., boca (bo); camisa, (cami)-. Se otorga 1 punto por cada respuesta correcta. Presenta de confiabilidad de 0.91.

c.2. Tareas que evalúan las distintas unidades fonológicas mientras se mantiene constante la dificultad de la tarea (Borzone y Signorini, comunicación personal, mayo 2005; Manrique y Gramigna, 1984).

5. Identificación de sonido Inicial. Esta prueba evalúa la capacidad para identificar y aparear sonidos. Se presenta al niño o niña una lámina con tres dibujos, uno en la parte superior de la hoja y dos en la parte inferior. El sonido inicial de uno de los dibujos de la parte inferior coincide con el sonido inicial del dibujo ubicado en la parte superior de la lámina. La persona menor debe identificar, entre los dos dibujos localizados en el sector inferior de la hoja, cuál es el que comienza con el mismo sonido que el dibujo ubicado en la parte superior. Primero se presentan tres ítems de ensayo corrigiendo las respuestas incorrectas y explicando por qué son incorrectas. Luego, se presentan los ítems de prueba. La prueba, con una confiabilidad de 0.80 , está constituida por un total de 10 ítems y se asigna 1 punto por cada respuesta correcta.

6. Identificación de sílaba inicial. Esta prueba evalúa la capacidad para identificar y aparear sílabas iniciales. Se presenta al niño una lámina con tres dibujos, uno en la parte superior de la hoja y dos en la parte inferior. La sílaba inicial de sólo uno de los dibujos de la parte inferior coincide con la silaba inicial del dibujo ubicado en la parte superior. El niño debe identificar, entre los dos dibujos del sector inferior de la hoja, cuál es el que comienza con la misma sílaba que el dibujo ubicado en la parte superior.

El procedimiento de aplicación es similar al de la prueba de identificación de sonido inicial: se dan ejemplos y contraejemplos y se toman 3 ítems de ensayo. La prueba está conformada por un total de 10 ítems. Se asigna 1 punto por cada respuesta correcta.

7. Identificación de sílaba final. El material de evaluación es similar al de las dos pruebas anteriores, pero la sílaba final del dibujo de la parte superior de la hoja coincide con la sílaba final de sólo uno de los dibujos de la parte inferior. El procedimiento de aplicación es igual al de las dos pruebas precedentes: se dan ejemplos y contraejemplos y se administran 3 ítems de ensayo. La prueba consta de 10 ítems y se otorga 1 punto por cada respuesta correcta. 


\subsubsection{Medición de las Variables Ambientales}

1. Nivel socioeconómico. Variable construida a partir de la combinación de la ocupación de los padres de los niños con el número de años de escolaridad aprobados (Instituto Nacional de Estadísticas y Censos [INDEC], 2008). Se distinguieron seis categorías de situación socioeconómica en el hogar otorgándole la siguiente puntuación: (1) marginal; (2) bajo inferior; (3) operario; (4) técnico; (5) técnico profesional y (6) profesional 2. Nivel educativo en el hogar. Según el INDEC (2008), el nivel educativo en el hogar es una variable conformada a partir del promedio de años aprobados de escolaridad por los miembros de 25 años y más del hogar. El nivel de escolaridad de cada uno de los padres fue obtenido del registro de grado. En dicho registro, el nivel de escolaridad se categoriza de la siguientes forma: primaria incompleta, primaria completa, secundaria incompleta, secundaria completa, terciario incompleto, terciario completo, universitario incompleto y universitario completo. Por lo tanto, de acuerdo con las categorías establecidas por el INDEC, a estas categorías asignamos los siguientes números de años aprobados de escolaridad obteniendo así una variable numérica: Primaria incompleta: hasta 3.5 años; Primaria completa: hasta 7 años; Secundaria incompleta: hasta 9.5 años; Secundaria completa: hasta 12 años; Terciario incompleto: hasta 14.5 años; Terciario completo: hasta 15.99 años; Universitario incompleto: hasta 15.99 años; Universitario completo: hasta 19.99 años. Para la variable nivel educativo de los padres se calculó el promedio de años de escolaridad de ambos padres. Para obtener un dato sociodemográfico de la variable dicho promedio se clasificó en las siguientes categorías: 1) promedio inferior al número de años equivalentes al secundario: secundario incompleto; 2) promedio equivalente al número de años del secundario o inferior al correspondiente a un nivel superior: secundario completo y 3 ) promedio de años equivalente a un nivel terciario o universitario: superior.

\section{Resultados y Discusión}

Se efectuaron dos análisis de varianza por separado para evaluar el efecto de la zona escolar (zona urbana y urbano-marginal) sobre el nivel socioeconómico y el nivel educativo. Se empleó un nivel alfa de 0.05 para todos los tests estadísticos.

Nivel socioeconómico. Se observó un efecto significativo de la zona donde está ubicado el establecimiento escolar $(F(1,112)=20.2, p<0.0001)$ sobre el nivel socioeconómico de los padres de los niños evaluados. Los escolares de escuelas urbano- 
marginales $(\mathrm{M}=2.5, \mathrm{SE}=0.12)$ presentan un nivel socioeconómico significativamente inferior al de escuelas urbanas $(\mathrm{M}=3.3, \mathrm{SE}=0.12$, $\mathrm{t}(109)=4,5, \mathrm{p}=0.000)$ (Figura 1).

Nivel educativo. El análisis evidenció un efecto significativo de la zona escolar sobre el nivel socioeconómico de los hogares de donde provienen los escolares evaluados $(F(1,111)$ $=13.7, p=0.0003)$. El nivel educativo de los padres de escolares que asisten a escuelas urbano-marginales $(\mathrm{M}=9.3, \mathrm{SE}=0.4)$ resultó ser significativamente inferior al de los padres de escolares que asisten a escuelas urbanas $(\mathrm{M}=11.5, \mathrm{SE}=0.4 ; \mathrm{t}(109)=4.4, \mathrm{p}=0.000)$ (Figura 2).

A continuación se realizaron dos análisis de correlación múltiple. El primero dirigido a evaluar las relaciones existentes entre el nivel educativo y el nivel socioeconómico de los padres con las habilidades pre-lectoras del grupo de escolares. Y el segundo, se realizó tendiente a evaluar las mismas relaciones pero teniendo en cuenta la zona.

En el primer análisis de correlación se observan asociaciones significativas del nivel socioeconómico con el nivel de vocabulario $(r=0.3 ; p<0.01)$ y el constructo conocimiento del nombre y sonido de la letra $(r=0.3 ; p<0.01)$. Particularmente, con conocimiento del nombre de las letras $(r=0.3 ; p<0.01)$ así como también la habilidad para nombrar un objeto que empiece con una letra determinada $(r=0.3 ; p<0.01)$, no así con la habilidad para enunciar el sonido que una letra representa. Respecto del nivel educativo, se evidenciaron asociaciones positivas y significativas con el constructo conocimiento del nombre y sonido de la letra $(r=0.4 ; p<0.01)$ y dos de sus componentes: conocimiento del nombre de la letra $(r=$ $0.4 ; p<0.01)$ y nombrar un objeto que empiece con una letra determinada $(r=0.3 ; p<0.01)$. Asimismo se evidenció una correlación significativa con la habilidad para aislar sonido consonántico $(r=0.3 ; p<0.05)$ (Tabla 2).

En el segundo análisis, al evaluar las relaciones entre el nivel socioeconómico y las habilidades lingüísticas de escolares que asisten a escuelas urbanas, se observan correlaciones positivas y significativas entre el nivel socioeconómico y el nivel de vocabulario $(r=0.3 ; p=0.01)$, el constructo conocimiento del nombre y sonido de la letra $(r=0.3 ; p=$ $0.01)$, sonido de la letra $(r=0.4 ; p<0.001)$, aislar sílabas $(r=0.3 ; p=0.05)$ y aislar sonidos consonánticos $(r=0.3 ; p<0.05)$. Con respecto al nivel educativo se observaron relaciones positivas y significativas con el constructo conocimiento del nombre y sonido de la letra $(r=$ $0.4 ; p=0.01)$, aislar sílabas $(r=0.4 ; p<0.01)$ y asilar sonido consonántico $(r=0.4 ; p<0.01)$ (Tabla 3). Con respecto a las escuelas urbano-marginales no se observaron correlaciones positivas significativas entre el nivel socioeconómico y educativo en el hogar con ninguna de 
las variables lingüísticas evaluadas en los niños y las niñas. Por el contrario, se evidenciaron correlaciones negativas del nivel socioeconómico con la habilidad para identificar el sonido de las letras $(r=-0.3 ; p<0.01)$, integración de sonidos $(r=0.4 ; p<0.01)$, identificación de sílaba inicial $(r=0.4 ; p<0.01)$, rima $(r=0.5 ; p<0.01)$, contar sílabas en palabras $(r=0.5 ; p$ $<0.01)$, aislar sílabas $(r=0.4 ; p<0.01)$ y asilar sonidos consonánticos $(r=0.4 ; p<0.01)$. En el mismo sentido, el nivel educativo en el hogar correlacionó de manera negativa y significativa con la habilidad para identificar rimas $(r=0.4 ; p<0.01)$ y aislar sílabas $(r=0.4 ; p$ $<0.01$ ) (Tabla 4).

Debido a que no encontramos resultados semejantes entre ambos factores ambientales (nivel socioeconómico y nivel educativo) con las habilidades lingüísticas evaluadas teniendo en cuenta la zona nos preguntamos si la disminución en el número de correlaciones significativas en zonas urbano-marginales podía deberse al efecto del año de escolarización por el que habían transitado las personas menores pertenecientes a ambas salas de 5 años evaluadas durante su asistencia a sala de 4 años. Por este motivo realizamos un análisis de regresión por cada edad por separado considerando como predictoras las variables ambientales (nivel socioeconómico y nivel educativo) y como dependientes aquellas habilidades lingüísticas con las que correlacionaron de manera significativa (vocabulario, el constructo conocimiento del nombre y sonido de la letra y aislar sonido consonántico).

En los dos primeros análisis la variable dependiente fue el vocabulario y la independiente el nivel socioeconómico. Para los niños y las niñas de 4 años, el nivel socioeconómico explica el $5 \%\left(R^{2}\right.$ corregido $\left.=0.05\right)$ de la varianza en el nivel de vocabulario de manera significativa $(t=2.1 ; p=0.02)$ mientras que en los de 5 años el porcentaje fue del 0,09\%, no resultando una variable significativa. Respecto del constructo conocimiento del nombre y sonido de la letra, el nivel socioeconómico explica el $6 \%\left(\mathrm{R}^{2}\right.$ corregido $\left.=0.05\right)$ de la varianza a los 4 años $(t=2.2 ; p=0.02)$ y el $.04\left(R^{2}\right.$ corregido $\left.=0.004\right)$ a los 5 años.

En los cuatro análisis siguientes la variable predictiva fue el nivel educativo. Con respecto al constructo conocimiento del nombre y sonido de la letra el nivel educativo explicó el $13 \%$ de la varianza $(t=3.1 ; p=0.002)$ en escolares de 4 años y el $1 \%$ en escolares de 5 años sin resultar ser significativa. Respecto de la variable dependiente, aislar sonido consonántico, en niños y niñas de 4 años el nivel educativo explicó el $10 \%$ de la varianza $\left(\left(R^{2}\right.\right.$ corregido $\left.\left.=0.096\right) ;(t=3.1 ; p=0.002)\right)$ y a los 5 años el $0.02 \%\left(R^{2}\right.$ corregido $\left.=0.002\right)$ de manera no significativa $(t=3.1 ; p=0.002)$. 


\section{Conclusiones}

Los resultados corroboraron nuestras primeras dos hipótesis: existen diferencias significativas tanto en el nivel socioeconómico como educativo entre los hogares de las personas que asisten a escuelas urbanas con el de las que asisten a escuelas urbanomarginales. Si bien este resultado no evalúa la relación directa con las habilidades lingüísticas adquiridas por niñas y niños que se desarrollan en contextos con diferentes características, es de fundamental importancia ya que estudios dirigidos a evaluar el efecto de variables ambientales tales como el nivel socioeconómico y el nivel educativo del hogar en que crece el grupo de escolares evalúan infantes procedentes de escuelas ubicadas en distintas regiones (urbanas, urbano-marginales y rurales) asumiendo que tales diferencias ambientales efectivamente existen en ausencia de evidencia empírica (por ejemplo, Filippetti, 2012). Aunque Filippetti (2012) menciona los coeficientes socioeconómicos de las escuelas otorgados por el Ministerio de Educación en base a los ingresos de la familia categorizados como bueno el perteneciente a una escuela urbana y deficitario el correspondiente a una escuela periférica, hasta donde pudimos indagar no hallamos un análisis estadístico de dicha variable entre ambas escuelas.

Los resultados confirmaron parcialmente la tercera hipótesis. Si bien las correlaciones halladas oscilaron entre leves y moderadas, se observaron relaciones positivas y significativas entre las variables ambientales nivel socioeconómico y educativo con algunas de las habilidades lingüísticas evaluadas en el grupo de preescolares. Particularmente, ambas variables correlacionaron de manera significativa con el constructo conocimiento del nombre y sonido de la letra. Dentro de este constructo ambas variables contextuales se asociaron de manera significativa con dos de las habilidades que integran el constructo: el conocimiento del nombre de la letra y nombrar un objeto que comience con una letra específica, no así con la habilidad para identificar sonido inicial. En este sentido, la correlación hallada entre el nivel educativo y socioeconómico del hogar con el conocimiento del nombre y sonido de la letra reviste una importancia particular, ya que en el marco de la psicología cognitiva el aprendizaje inicial de la lectura se sustenta en dos procesos fundamentales: el del procesamiento fonológico y la adquisición del conocimiento alfabético (Caravolas, Hulme y Snowling, 2001). Aun más, con respecto al conocimiento alfabético, el estudio de Burgess, Hecht y Lonigan (2002) corrobora que las primeras nociones sobre el conocimiento de las letras se adquieren mediante las experiencias de enseñanza explícita que los adultos organizan para los niños en sus hogares. 
Al considerar las correlaciones en el contexto escolar (urbano y urbano-marginal) se observó que mientras que en las escuelas urbanas se mantienen las correlaciones entre las variables ambientales y las variables lingüísticas, en las escuelas urbano-marginales no se obtienen asociaciones significativas. En escuelas urbanas se evidencian asociaciones significativas entre el nivel socioeconómico y el nivel de vocabulario y el conocimiento y nombre de la letra por un lado, y entre el nivel educativo de los padres con el conocimiento del nombre y sonido de la letra, aislar sonidos y sílabas por el otro. En cambio, tales asociaciones no se presentan en el contexto de las escuelas urbano-marginales. A partir de lo expuesto y considerando que los datos obtenidos pertenecen a una muestra constituida por escolares de 4 y 5 años de edad, efectuamos diferentes análisis de regresión lineal para cada edad (4 y 5 años) entre las variables contextuales y aquellas habilidades lingüísticas que correlacionaron significativamente. Las asociaciones entre las variables ambientales y las lingüísticas sólo resultaron significativas para los escolares que asisten a salas de 4 años: a) el nivel socioeconómico resultó ser una variable predictiva tanto del nivel de vocabulario como del conocimiento del nombre y sonido de la letra y b) el nivel educativo de los padres del conocimiento del nombre y sonido de la letra y de aislar sonido consonántico. En coincidencia con los resultados de Burgess, Hecht y Lonigan (2002) las actividades literarias llevadas a cabo en el hogar contribuyen con la adquisición de nociones pre-lectoras, particularmente con el conocimiento del nombre y sonido de las letras. Posteriormente, la comprensión del principio alfabético se completa en la escuela mediante las experiencias de aprendizaje que las docentes diseñan para tal fin. Es decir que la ausencia de correlación entre las variables contextuales y las lingüísticas a la edad de 5 años podría deberse al efecto de las experiencias lingüísticas y literarias promovidas por los docentes durante el primer año de escolarización-sala de 4 años. Los resultados sugieren que aunque las variables contextuales tales como el nivel educativo de los padres o el socioeconómico pueden explicar cierto porcentaje en la varianza de habilidades lingüísticas consideradas predictoras del posterior rendimiento lector, la escuela es una variable interviniente que regula tales efectos.

Los resultados adquieren sentido en la interpretación del modelo de adquisición de la lectura propuesto por Diuk y Ferroni (2011) quienes consideran que, aunque las variables contextuales dan cuenta de las variaciones en ciertas habilidades lingüísticas específicamente en el conocimiento del nombre y sonido de las letras-, las mismas resultan insuficientes para explicar la variación de tal habilidad en su totalidad. Las autoras explican 
que existen además diferencias en el aprendizaje de dicha habilidad asociadas a la sensibilidad fonológica, una sensibilidad propia que presentan los niños y las niñas para desarrollar ciertas habilidades (Diuk y Ferroni, 2011, p. 73). Aquí además nos parece oportuno destacar el rol protector que ejerce el sistema educativo mediante la incorporación de salas de 4 años al Nivel Inicial con actividades curriculares dirigidas a fortalecer, encauzar y promover en los pequeños escolares el bagaje de habilidades y recursos cognitivos y lingüísticos que presentan al iniciar su recorrido en el ámbito escolar.

Los resultados, además de demostrar la relación entre el nivel socioeconómico y el nivel educativo del hogar con la adquisición de habilidades lingüísticas indican que la educación inicial recibida en el contexto escolar es un ámbito privilegiado que, frente a contextos socio-económicos heterogéneos minimiza la inequidad promoviendo los recursos cognitivos de los escolares mediante estrategias pedagógicas específicas diseñadas para facilitar la adquisición de habilidades académicas tales como la conciencia fonológica que, de manera indirecta lleva a los niños y las niñas a reflexionar sobre los sonidos iniciales de sus nombres y las letras, habilidad considerada de manera indiscutible precursora del aprendizaje de la lecto-escritura.

\section{Referencias}

Adams, Marilyn, Foorman, Barbara; Lundberg, Ingvar y Beeler, Terri. (2003). Phonemic awareness in young children. Baltimore, Maryland: Brookes Publishing.

Anderson, Richard y Freebody, Peter. (1981). Vocabulary Knowledge: En John T. Guthrie (Ed.), Comprehension and teaching: Research and reviews (pp. 77-116). Newark, DE: International Reading Association.

Baker, Doris, Al Otaiba, Stephanie, Ortiz, Miriam, Correa, Vivian y Cole, Ron. (2014). Vocabulary development and intervention for English Language Learners in the early grades. In Janette B. Benson (Ed.), Advances in Child Development and Behavior (vol. 46, pp. 281-338). San Diego, CA: Elsevier.

Benítez, Yolanda, Vargas Gustavo, Hernández Alfredo, Sánchez Ulises y García Ángela. (2007). Habilidades lingüísticas en niños de estrato sociocultural bajo, al iniciar la primaria. Acta Colombiana de Psicología, 10(2), 9-17.

Borzone, Ana María, Rosemberg, Celia, Diuk, Beatriz y Amado, Bibiana. (2005). Aprender a leer y escribir en contextos de pobreza: Una propuesta de alfabetización intercultural. Lingüística en el Aula, 8, 7-27.

Bradley, Lynette y Bryant, Peter. (1983). Categorizing sounds and learning to read. A causal connection. Nature, 301, 419-421. 
Bravo Valdivieso, Luis. (2000). Los procesos cognitivos en el aprendizaje de la lectura inicial. Pensamiento Educativo, 27, 49-68.

Bronfenbrenner, Urie (1995). Developmental Ecology through space and time: A future perspective. En Phyllis Moen, Glenn H. Elder y Kurt Lüscher (Eds.), Examining lives in contexto: Perspectives on the ecology of human development (pp. 619-647). Washington, D.C.: American Psychoogical Association.

Buckner, Jhon, Bassuk, Ellen y Weinreb, Linda. (2001). Predictors of academic achievement among homeless and low-income housed children. Journal of School Psychology, 39(1), 45-69.

Burgess, Stephen, Hecht, Steven y Lonigan, Christopher. (2002). Relations of the home literacy environment (HLE) to the development of reading-related abilities: A one-year longitudinal study. Reading Research Quarterly, 37(4), 408-426.

Caravolas, Markéta, Hulme, Charles y Snowling, Margaret. (2001). The foundations of spelling ability: Evidence from a 3-year longitudinal study. Journal of Memory and Language, 45(4), 751-774.

Dearing, Eric, McCartney, Katheleen, Weiss, Holly, Kreider y Simpkins, Sandra. (2004). The promotive effects of family educational involvement for low-income children's literacy. Journal of School Psychology, 42(6), 445-460.

Dickinson, David. (2012). Teachers' language practices and academic outcomes of preschool children. Science, 333(6045), 964-967.

Dickinson, David y Tabors, Patton. (2001). Beginning literacy with language. Young children learning at home and school. Baltimore: Paul H. Brookes Publishing.

Dickinson, David, Anastasopoulos, Louisa, McCabe, Allyssa, Peisner-Feinberg, Ellen y Poe, Michele. (2003). The comprehensive language approach to early literacy: The interrelationships among vocabulary, phonological sensitivity and print knowledge among preschool-aged children. Journal of Educational Psychology, 95(3), 465-481.

Dickinson, David y Caswell, Linda. (2007). Building support for language and early literacy in preschool classrooms through in-service professional development: Effects of the literacy environment enrichment program (LEEP). Early Childhood Research Quarterly, 22(2), 234-260.

Diuk, Beatriz y Ferroni, Marina. (2011). Adquisición lectora en niños que crecen en contextos de pobreza: perfiles cognitivos de niños con alto y bajo nivel lector. Cuadernos de Psicopedagogía, 7, 70-89.

Ehri, Linnea, Nunes, Simone, Willows, Dale, Shcuster, Barbara, Yaghoub-Zadeh, Zoreh y Shanahan, Timothy. (2001). Phonemic awareness instruction helps children learn to read. Evidence from the National Reading Panel's meta-analysis. Reading Research Quarterly, 36(3), 250-287. 
Fernández Cano, Antonio, Machuca, Mariano y Lorite, Javier. (2002). Discriminancia de habilidades metalingüísticas segmentarias sobre el español hablado. Un estudio comparativo de buenos frente a pobres lectores. Revista Española de Pedagogía, 60(221), 147-169.

Filippetti, Vanessa. (2012). Estrato Socioeconómico y Habilidades Cognitivas en Niños Escolarizados: Variables Predictoras y Mediadoras. Psykhe, 21(1), 3-20.

Hart, Betty y Risley, Todd. (1995). Meaningful differences in the everyday experience of young American children. Baltimore: Paul $\mathrm{H}$. Brookes Publishing.

Hoff, Erika. (2003). The specificity of environmental influence: socioeconomic status affects early vocabulary development via maternal speech. Child Development, 74(5), 13681378.

Hoff, Erika y Tian Chunyan. (2005). Socioeconomic status and cultural influences on language. Journal of Communication Disorders, 38(4), 271-278.

Instituto Nacional de Estadísticas y Censos. (2008). Sistema de Estadísticas Sociodemográficas. Área Educación. Definiciones y conceptos. Recuperado de http://www.indec.mecon.ar/nuevaweb/cuadros/7/definiciones sesd educacion.pdf

Jadue, Gladys. (1997). Factores ambientales que afectan el rendimiento escolar de los niños provenientes de bajo nivel socioeconómico y cultural. Estudios psicopedagógicos, (23), 75-80. Doi: 10.4067/S0718-07051997000100007

Jiménez Juan y Ortiz, María del Rosario. (1995). Conciencia fonológica y aprendizaje de la lectura: Teoría, Evaluación e Intervención. Madrid: Síntesis.

Manrique, Ana María y Gramigna, Susana. (1984). La segmentación fonológica y silábica en niños de preescolar y primer grado. Lectura y Vida, 5(1), 4-13.

Muter, Val, Hulme, Charles, Snowling, Margaret y Taylor, Sydney. (1998). Segmentation, not rhyming, predicts early progress in learning to read. Journal of Experimental Child Psychology, 71(1), 3-27.

Pennington, Bruce y Lefly, Dianne. (2001). Early Reading Development in Children at Family Risk for Dyslexia. Child Development, 72(3), 816-833.

Porta, María Elsa. (2008). Hacia un enfoque comprehensivo del aprendizaje lingüístico inicial. Implementación del modelo y evaluación de sus efectos sobre el rendimiento lector. Mendoza: Universidad Nacional de Cuyo.

Porta, María Elsa e Ison, Mirta. (2011). Hacia un enfoque comprensivo del aprendizaje lingüístico inicial como proceso cognitivo. Revista Iberoamericana de Educación, (55), 243-260.

Porta, María Elsa y Difabio, Hilda Emilia. (2011). Identificación de predictores del Aprendizaje lingüístico Inicial: Efecto de factores cognitivos, lingüísticos y ambientales. Psico/Pedagógica, 12, 70-93. 
Porta, María Elsa, Harper, Lawrence y Kraft, Rosemarie. (2010). Hemispheric asymmetry profiles during beginning reading. Effects of reading level and word type. Developmental Neuropsychology, 35(1), 96-114.

Share, David, Jorm, Anthony, MacLean, Rod y Mathews, Russell. (1984). Sources of individual differences in reading acquisition. Journal of Educational Psychology, 76(6), 1309-1324.

UNESCO. (2007). Literacy Public Report from de UNESCO. Institute for Statistics. Recuperado de http://data.uis.unesco.org/index.aspx?queryid=166\#

UNICEF. (2007). Propuestas para Superar el Fracaso Escolar. Buenos Aires: Unicef.

Verhoeven, Ludo, van Leeuwe, Jan y Vermeer, Anne. (2011). Vocabulary growth and reading development across the elementary school years. Scientific Studies of Reading, 15(1), 8-25.

Wechsler, David. (2004). Test de inteligencia para preescolares (WPPSI) Manual (3ª ed.). Buenos Aires: Paidós.

Woodcock, Richard y Muñoz-Sandoval, Ana. (1996). Batería estándar. Pruebas de aprovechamiento revisada. US: Riverside Publishing.

Yopp, Hallie. (1988). The validity and reliability of phonemic awareness tests. Reading Research Quarterly, 23(2), 159-177.

Ziegler, Johannes y Goswami, Usha. (2005). Reading acquisition, developmental dislexia, and skilled reading across languages: a psycholinguistic grain size theory. Psychological Bulletin, 131(1), 3-29. 
Tabla 1. Tabla de variables socio-demográficas

\begin{tabular}{|c|c|c|c|c|}
\hline & \multicolumn{2}{|c|}{ Sala de 4 años } & \multicolumn{2}{|c|}{ Sala de 5 años } \\
\hline & EU & EUM & EU & EUM \\
\hline No. de varones & 9 & 15 & 14 & 13 \\
\hline No. de niñas & 21 & 15 & 11 & 13 \\
\hline Edad media (meses) & 63 & 63 & 77 & 77 \\
\hline Nivel de lectura (grado) & $410(\mathrm{k} .7)$ & $410(\mathrm{k} .7)$ & 439 (k.8) & 427 (k.7) \\
\hline \multicolumn{5}{|l|}{ Nivel educativo familiar } \\
\hline Secundario incompleto & 8 & 19 & 9 & 15 \\
\hline Secundario completo & 20 & 10 & 21 & 15 \\
\hline Superior & 1 & 0 & 0 & 0 \\
\hline \multicolumn{5}{|l|}{ Nivel Socioeconómico } \\
\hline Marginal & 0 & 2 & 2 & 6 \\
\hline Bajo Inferior & 3 & 4 & 2 & 13 \\
\hline Operario sin calificación & 14 & 21 & 17 & 6 \\
\hline Técnico & 10 & 1 & 9 & 1 \\
\hline Técnico Profesional & 1 & 0 & 0 & 0 \\
\hline Profesional & 2 & 0 & 0 & 0 \\
\hline
\end{tabular}


Figura 1

Efecto de la Zona sobre el Nivel Socioecómico

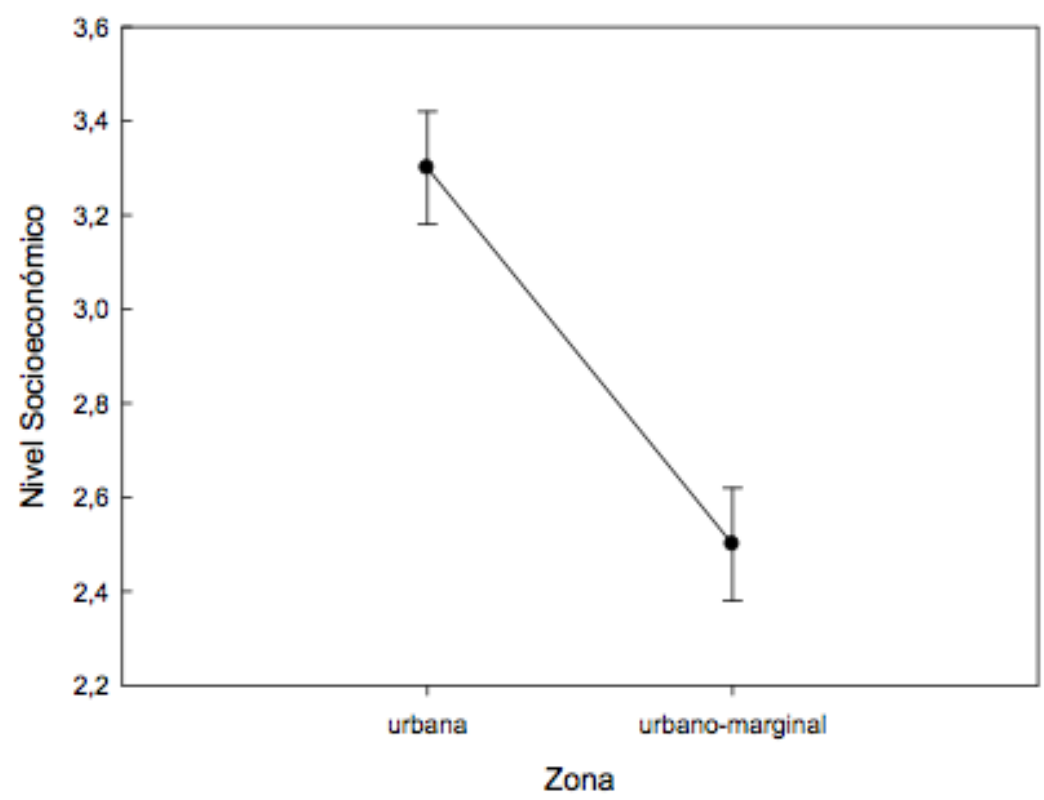

Figura 2

Efecto de la Zona sobre el Nivel Educativo

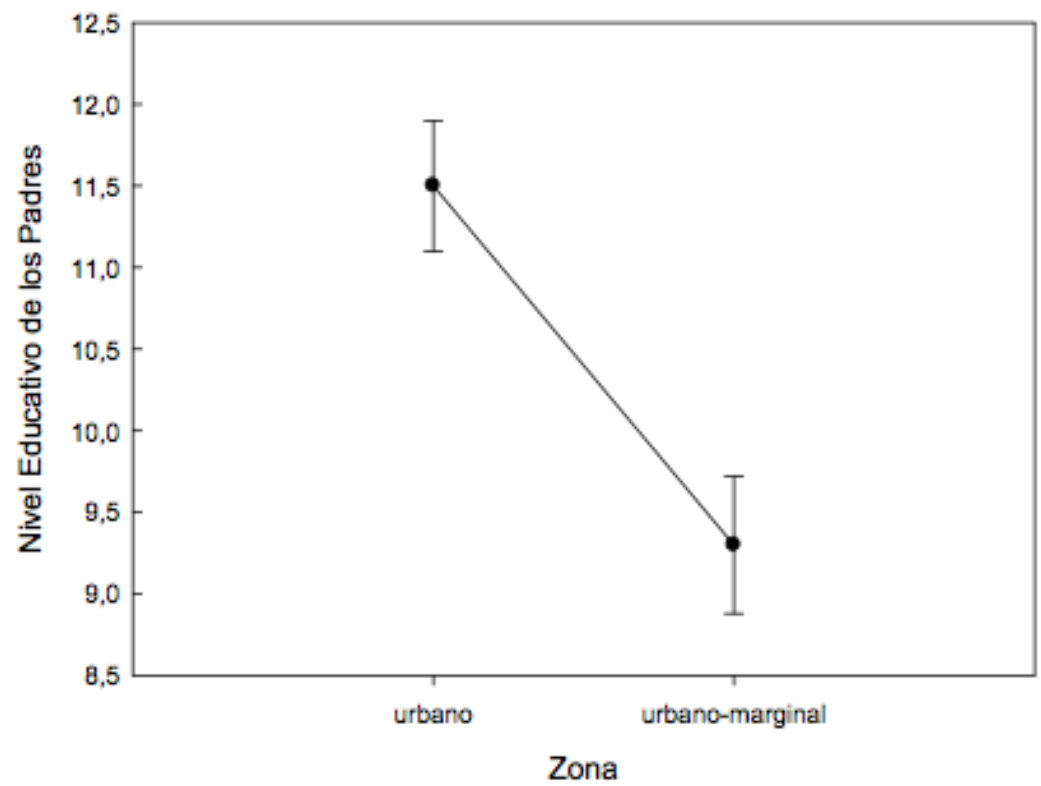


Tabla 2. Correlaciones entre variables contextuales y variables lingüísticas

\begin{tabular}{|c|c|c|c|c|c|c|c|c|c|c|c|c|c|c|c|}
\hline & 1 & 2 & 3 & 4 & 5 & 6 & 7 & 8 & 9 & 10 & 11 & 12 & 13 & 14 & 15 \\
\hline $\begin{array}{l}\text { 1.Nivel } \\
\text { Socioeconómico }\end{array}$ & 1,000 & & & & & & & & & & & & & & \\
\hline 2. Nivel Educativo & 0,711 & 1,000 & & & & & & & & & & & & & \\
\hline 3.Vocabulario & $0,276^{* *}$ & 0,146 & 1,000 & & & & & & & & & & & & \\
\hline $\begin{array}{l}\text { 4.Conoc de las } \\
\text { letras }\end{array}$ & $0,340^{*}$ & 0,384 & $0,254^{*}$ & 1,000 & & & & & & & & & & & \\
\hline 5. Nombre & $0.323^{* *}$ & $0,352^{* * *}$ & $0,261^{* *}$ & $0,964^{* * *}$ & 1,000 & & & & & & & & & & \\
\hline 6. Sonido & 0,197 & 0,330 & 0,181 & $0,553^{* * *}$ & $0,427^{\cdots *}$ & 1,000 & & & & & & & & & \\
\hline 7. Es la de & $0,329^{* *}$ & $0,357^{* * *}$ & 0,214 & $0,962 *$ & $0,880^{n * *}$ & $0,458^{* * *}$ & 1,000 & & & & & & & & \\
\hline $\begin{array}{l}\text { 8. Integración de } \\
\text { sonidos }\end{array}$ & $-0,065$ & 0,067 & 0,184 & $0,336 *$ & $0,294^{*}$ & $0,327^{* *}$ & $0,318^{*}$ & 1,000 & & & & & & & \\
\hline $\begin{array}{l}\text { 9. Identificar el } \\
\text { sonido inicial }\end{array}$ & 0,134 & 0,194 & $0,262^{*}$ & $0,329^{* * *}$ & $0,305^{* *}$ & $0,225^{*}$ & $0,318^{* * *}$ & 0,265 & 1.00 & & & & & & \\
\hline $\begin{array}{l}\text { 10. Identificar } \\
\text { sílaba inicial }\end{array}$ & $-0,040$ & $-0,089$ & 0,124 & $0,348^{\cdots *}$ & $0,324^{* *}$ & $0,089^{*}$ & $0,377^{\cdots *}$ & $0,296^{*}$ & $0,521^{\cdots *}$ & 1,000 & & & & & \\
\hline 11. Identificar rima & 0,004 & 0,045 & $0,322^{* * *}$ & $0,362^{* * *}$ & $0,331^{* *}$ & $0,247^{*}$ & $0,355^{* *}$ & $0,445^{* *}$ & $0,427^{* * *}$ & $0,539^{* * *}$ & 1,000 & & & & \\
\hline $\begin{array}{l}\text { 12. Contar sílabas } \\
\text { en palabras }\end{array}$ & 0,109 & 0,128 & 0,356 & $0,544^{* *}$ & $0,479^{* * *}$ & $0,347^{* * *}$ & $0,562 *$ & $0,551^{* *}$ & $0.343^{* * *}$ & $0,391^{* \cdots}$ & 0,520 & 1,000 & & & \\
\hline 13. Aislar Sílabas & 0,074 & 0,161 & $0,439^{* *}$ & $0,471^{\cdots *}$ & $0,416^{* *}$ & $0,421^{* * *}$ & $0,452^{\cdots *}$ & $0,389^{* * *}$ & $0,394^{* * *}$ & $0,283^{* \cdots}$ & $0,495^{* * *}$ & $\cdots$ & 1,000 & & \\
\hline $\begin{array}{l}\text { 14. Aislar sonido } \\
\text { consonántico }\end{array}$ & 0,158 & $0,342^{*}$ & 0,183 & $0,370^{* *}$ & $0,350^{* *}$ & 0,195 & $0,367^{* *}$ & $0,385^{* *}$ & $0,523^{* * *}$ & $0,335^{* \cdots}$ & $0,360^{* * *}$ & $\cdots$ & $0,479^{* * *}$ & 1,000 & \\
\hline $\begin{array}{l}\text { 15. Omisión de } \\
\text { sílabas y fonemas }\end{array}$ & 0,1043 & 0,122 & $0,327^{* * *}$ & $0,487^{\cdots *}$ & $0,4666^{* * *}$ & $0,168^{*}$ & $0,500^{* \cdots}$ & $0,425^{*}$ & 0,426 "** & $0,579^{* *}$ & $0,465^{* *}$ & $\cdots$ & $0,421^{\cdots *}$ & $0,378^{* * *}$ & 1,000 \\
\hline
\end{tabular}

$p<0.05,{ }^{* *} p<0.01,{ }^{* * *} p<0.0001$ 
Tabla 3. Correlación entre variables ambientales y lingüísticas en zonas urbanas

\begin{tabular}{|c|c|c|c|c|c|c|c|c|c|c|c|c|c|c|c|}
\hline & 1 & 2 & 3 & 4 & 5 & 6 & 7 & 8 & 9 & 10 & 11 & 12 & 13 & 14 & 15 \\
\hline $\begin{array}{l}\text { 1.Nivel } \\
\text { Socioeconómico }\end{array}$ & 1,000 & & & & & & & & & & & & & & \\
\hline 2. Nivel Educativo & $0,73^{* * *}$ & 1,000 & & & & & & & & & & & & & \\
\hline 3.Vocabulario & $0,324^{* *}$ & 0,204 & 1,000 & & & & & & & & & & & & \\
\hline $\begin{array}{l}\text { 4.Nombre y sonido } \\
\text { de letras }\end{array}$ & $0,320^{*}$ & $0,398^{* *}$ & $0,2981^{*}$ & 1,000 & & & & & & & & & & & \\
\hline 5. Nombre & $0,277^{*}$ & $0,344^{* *}$ & $0,276^{*}$ & $0,967^{* * *}$ & 1,000 & & & & & & & & & & \\
\hline 6. Sonido & $0,439^{* * *}$ & $0,491^{* * *}$ & $0,295^{*}$ & $0,599^{* * *}$ & $0.478^{* * *}$ & 1,000 & & & & & & & & & \\
\hline 7. Es la de & $0,283^{*}$ & $0,367^{* *}$ & 0,2721 & $0,966^{* * *}$ & $0,888^{* * *}$ & $0,532^{* * * *}$ & 1,000 & & & & & & & & \\
\hline $\begin{array}{l}\text { 8. Integración de } \\
\text { sonidos }\end{array}$ & 0,111 & 0,190 & 0,130 & $0,444^{* * *}$ & $0,398^{* *}$ & $0,330^{\star}$ & $0,450^{* * * *}$ & 1,000 & & & & & & & \\
\hline $\begin{array}{l}\text { 9. Identificar el } \\
\text { sonido inicial }\end{array}$ & 0,196 & 0,208 & $0.437^{+* *+}$ & $0,307^{\star}$ & $0,293^{*}$ & 0,206 & $0,296^{*}$ & $0,367^{* *}$ & 1,000 & & & & & & \\
\hline $\begin{array}{l}10 . \quad \text { Identificar } \\
\text { sílaba inicial }\end{array}$ & $-0,000$ & $-0,130$ & 0,183 & $0,317^{* *}$ & $0,321^{* *}$ & $-0,032$ & $0,347^{* *}$ & $0,340^{* *}$ & $0,517^{* * *}$ & 1,000 & & & & & \\
\hline 11. Identificar rima & 0,078 & 0,106 & $0.323^{*}$ & $0,333^{* *}$ & $0,330^{* *}$ & 0,141 & $0,327^{* *}$ & $0,426^{* * *}$ & $0,458^{* * *}$ & $0,483^{* * *}$ & 1,000 & & & & \\
\hline 12. Contar sílabas & 0,259 & 0,217 & $0,394^{* *}$ & $0,615^{* * *}$ & $0,554^{* * *}$ & $0,345^{* *}$ & $0,648^{* * * *}$ & $0,539^{* * *}$ & $0,340^{* *}$ & $0,354^{* *}$ & $0,362^{* *}$ & 1,000 & & & \\
\hline 13. Aislar Sílabas & 0,275 & $0,368^{* *}$ & $0,465^{* *}$ & $0,611^{* * *}$ & $0,558^{* * *}$ & $0,509^{* * *}$ & $0,592^{* * *}$ & $0,392^{* *}$ & $0,451^{* * *}$ & $0,284^{* * *}$ & 0,402 & $0,534^{* * *}$ & 1,000 & & \\
\hline $\begin{array}{l}\text { 14. Aislar sonido } \\
\text { consonántico }\end{array}$ & $0,291^{*}$ & $0,439^{* *}$ & 0,167 & $0,406^{* *}$ & $0,390^{* *}$ & 0,125 & $0,425^{* * *}$ & $0,408^{* *}$ & $0,625^{* * *}$ & $0,348^{* *}$ & 0,239 & $0.524^{* * *}$ & $0,472^{* * *}$ & 1,000 & \\
\hline $\begin{array}{l}\text { 15. Omisión de } \\
\text { sílabas y fonemas }\end{array}$ & 0,147 & 0,183 & $0,429^{* * * *}$ & $0,552^{* * *}$ & $0,536^{* * *}$ & 0,169 & $0,574^{* * * *}$ & $0,483^{* * *}$ & $0,471^{* * *}$ & $0,541^{* * *}$ & $0,483^{* *}$ & $0,581^{* * *}$ & $0,538^{* * *}$ & $0,486^{\star \star \star}$ & 1,000 \\
\hline
\end{tabular}

Zona Urbana. . ${ }^{*} p<0.05,{ }^{* *} p<0.01,{ }^{* * *} p<0.001$ 
Tabla 4. Correlación entre variables ambientales y lingüísticas en zonas urbano-marginales

\begin{tabular}{|c|c|c|c|c|c|c|c|c|c|c|c|c|c|c|c|}
\hline & 1 & 2 & 3 & 4 & 5 & 6 & 7 & 8 & 9 & 10 & 11 & 12 & 13 & 14 & 15 \\
\hline $\begin{array}{l}\text { 1.Nivel } \\
\text { Socioeconómico }\end{array}$ & 1,000 & & & & & & & & & & & & & & \\
\hline 2. Nivel Educativo & $0,405^{\star \star}$ & 1,000 & & & & & & & & & & & & & \\
\hline 3.Vocabulario & 0,021 & $-0,209$ & 1,000 & & & & & & & & & & & & \\
\hline $\begin{array}{l}\text { 4.Nombre y sonido } \\
\text { de letras }\end{array}$ & $-0,030$ & $-0,109$ & $-0,082$ & 1,000 & & & & & & & & & & & \\
\hline 5. Nombre & 0.133 & $-0,009$ & 0,102 & $0,906^{\star \star *}$ & 1,000 & & & & & & & & & & \\
\hline 6. Sonido & $-0,324^{* *}$ & $-0,018$ & $-0,103$ & $0,684^{* \star *}$ & $0,501^{* * *}$ & 1,000 & & & & & & & & & \\
\hline 7. Es la de & $-0,024$ & $-0,212$ & $-0,217$ & $0,903^{* \star *}$ & 0,710 & $0,458^{\star \star}$ & 1,000 & & & & & & & & \\
\hline $\begin{array}{l}\text { 8. Integración de } \\
\text { sonidos }\end{array}$ & $-0,438^{\star *}$ & $-0,173$ & $0,368^{* *}$ & 0,129 & 0,069 & $0,306^{\star *}$ & 0,049 & 1,000 & & & & & & & \\
\hline $\begin{array}{l}\text { 9. Identificar el } \\
\text { sonido inicial }\end{array}$ & $-0,170$ & 0,079 & $-0,277$ & $0,460^{\star \star *}$ & 0.401 & 0,301 & $0,439^{* * *}$ & 0,058 & 1.000 & & & & & & \\
\hline $\begin{array}{l}\text { 10. Identificar } \\
\text { sílaba inicial }\end{array}$ & $-0,394$ & $-0,165$ & $-0,056$ & $0,505^{\star \star \star}$ & $0,365^{\star *}$ & $0,390^{\star *}$ & $0,519^{\star \star \star}$ & 0,252 & $0,522^{* \star *}$ & 1.000 & & & & & \\
\hline 11. Identificar rima & $-0,520^{\star \star \star}$ & $-0,375^{\star *}$ & $0,286^{*}$ & $0,456^{\star \star \star}$ & $0,298^{\star}$ & $0,544^{\star \star \star}$ & $0,402^{\star \star \star}$ & $0,555^{\star \star \star}$ & $0,334^{\star *}$ & $0,625^{\star \star \star}$ & 1.000 & & & & \\
\hline 12. Contar sílabas & $-0,488^{* *}$ & $-0,279$ & 0,235 & $0,312^{* *}$ & 0,188 & $0,392^{\star \star}$ & 0,279 & $0,629^{* \star *}$ & $0,330^{* *}$ & $0,439^{\star \star *}$ & $0,821^{* * *}$ & 1.000 & & & \\
\hline 13. Aislar Sílabas & $-0,416^{* *}$ & $-0,393^{* *}$ & $0,400^{* *}$ & 0,102 & $-0,027$ & $0,238^{*}$ & 0,121 & $0,385^{* *}$ & $0,280^{*}$ & $0,291^{*}$ & $0,706^{\star * *}$ & $0.635^{\star \star \star}$ & 1,000 & & \\
\hline $\begin{array}{l}\text { 14. Aislar sonido } \\
\text { consonántico }\end{array}$ & $-0,366^{* *}$ & $-0,022$ & 0,197 & 0,215 & 0,165 & $0,393^{\star *}$ & 0,096 & $0,379^{* *}$ & 0,260 & $0,282^{*}$ & $0,588^{* * *}$ & $0,469^{\star \star \star}$ & $0,507^{\star \star \star}$ & 1.000 & \\
\hline $\begin{array}{l}\text { 15. Omisión de } \\
\text { sílabas y fonemas }\end{array}$ & $-0,211$ & $-0,229$ & 0,018 & 0,197 & 0,134 & 0,203 & 0,184 & $0,347^{\star \star *}$ & $0,290^{*}$ & $0,644^{\star \star \star}$ & $0,392^{\star *}$ & 0,172 & 0,193 & 0,101 & 1.000 \\
\hline
\end{tabular}

Zona Urbano Marginal. ${ }^{*} p<0.05,{ }^{* *} p<0.01,{ }^{* *} p<0.001$ 